KYUNGPOOK Math. J. 53(2013), 105-116

http://dx.doi.org/10.5666/KMJ.2013.53.1.105

\title{
On the Folding of the Rectangular Distribution
}

\author{
S. A. El-SheHAWY* ${ }^{* \dagger}$ AND M. BASHeR ${ }^{\dagger}$ \\ Department of Mathematics, Faculty of Science, Menoufia University, Shebin El- \\ Kom, Egypt \\ and \\ Department of Mathematics, Faculty of Science, Suez Canal University, Egypt \\ e-mail : shshehawy64@yahoo.com and m_e_basher@yahoo.com
}

Abstract. Based on the concept of the folding, the folding in X-direction and in Ydirection are defined and denoted by the X-Folding and the Y-Folding respectively. We consider a random variable $X$ which follows a rectangular distribution " $R(a, b)$ distribution" with two parameters $a, b$. This paper aims to apply the folding on the unit area $P(a \leq X \leq b)$ and also to study the proposed folding in each direction for $R(a, b)$ distribution and the generated family of the corresponding constructed rectangular probability distributions. Some main properties of this family are reviewed. According to the proposed folding, we derive and discuss some important corresponding functions in closed forms.

\section{Introduction}

The main idea of folding on manifolds has been introduced by S. A. Robertson who studied the stratification determined by the folds or singularities [13]. Based on this paper, some studies on folding in many branches such as manifolds, topology, graphs-theory and algebra have been presented in some literatures $[1,2,3,4,5,6$, $7,8,9,12]$. The conditional foldings of manifolds have been defined by M. El-Ghoul [3]. Some applications on the folding of a manifold into itself were introduced by P. Di. Francesco [2]. Also a graph folding has been presented and discussed by E. El-Kholy [7]. Moreover, the theory of isometric foldings has been pushed and also some different types of foldings have been discussed by E. El-Kholy and others [9]. In this article we will discuss the folding of a family of probability distributions into a generated family of the same type of the probability distributions. Our study will

${ }^{*}$ Corresponding Author.

${ }^{\dagger}$ Current Address: Department of Mathematics, College of Science, Qassim University, P.O. Box 6644 Buraidah 51452, KSA.

Received October 25, 2011; accepted September 17, 2012.

2010 Mathematics Subject Classification: 57N10, 60E05.

Key words and phrases: Rectangular distribution, Folding.

This work was financially supported by the Deanship of Scientific Research at Qassim University, KSA, Project No. 1612. 
be concerned on the folding of the rectangular distribution $R(a, b)$ with two parameters $a, b$, where $a<b$. We will apply the X-Folding and Y-Folding, which generate a sequence of areas and a corresponding sequence of rectangular distributions. Some fundamental properties of the $R(a, b)$ distribution are reviewed in $[10,11]$. Thus, we will be concerned on a study of some main properties of the generated family of rectangular distributions by folding.

This paper is organized as follows: Section 2 reviews the original $R(a, b)$ distribution and its fundamental properties. In sections 3 and 4 , we introduce the $\mathrm{X}$-Folding and the Y-Folding and present a study of the generated families of the corresponding rectangular probability distributions and some main statistical and geometrical properties. Section 5 gives some attention to application. We summarize the results and give some features and comments in section 6.

\section{The rectangular distribution and its properties}

In probability theory and statistics, the rectangular distribution is a family of probability distributions such that for each member of the family, all intervals of the same length on the distribution's support are equally probable. The support is defined by the two parameters $a, b$, which are its minimum and maximum values (start-point and end-point). The distribution is often abbreviated $R(a, b)$.

According to $[10,11]$, the continuous random variable $X$ has a $R(a, b)$ distribution if its probability density function is given by:

$$
g_{R(a, b)}(x)=\left\{\begin{array}{clc}
\frac{1}{b-a} & : & x \in[a, b] \\
0 & : & \text { otherwise }
\end{array}\right.
$$

The cumulative distribution function of the $R(a, b)$ variable $X$ is

$$
G_{R(a, b)}(x)=\left\{\begin{array}{clc}
0 & : & x \leq a \\
\frac{x-a}{b-a} & : & a<x<b \\
1 & : & x \geq b
\end{array}\right.
$$

with the corresponding inverse cumulative distribution function (critical value or quantile function)

$$
x_{\alpha}^{R(a, b)}=G_{R(a, b)}^{-1}(\alpha)=a+\alpha(b-a),
$$

where $P\left[X>x_{\alpha}^{R(a, b)}\right]=\alpha, \alpha \in(0,1)$. The $R(a, b)$ distribution is symmetric about the mean $\mu=(a+b) / 2$ and its variance $\sigma^{2}$ equals $(b-a)^{2} / 12$. It has zero value for the skewness and its excess kurtosis is equal to -1.20 . Moreover, its momentgenerating function and the characteristic function are given respectively as the 
following:

$$
M_{R(a, b)}(t)=\frac{e^{b t}-e^{a t}}{(b-a) t}, \quad \Psi_{R(a, b)}(t)=\frac{e^{i b t}-e^{i a t}}{i(b-a) t}, \quad|t| \neq 0, \quad i=\sqrt{-1} .
$$

From the moment generating function, the r-th non-central moments can be calculated by the closed form $\mu_{r}^{\prime}=\frac{1}{r+1} \sum_{k=0}^{r} a^{k} b^{r-k}$. The probability that a rectangular (uniformly) distributed random variable fall within any interval of fixed length is independent of the location of the interval itself (but it is dependent on the interval size), so long as the interval is contained in the distribution's support. To see this, if $X$ has $R(a, b)$ distribution and $[x, x+d]$ is a subinterval of $[a, b]$ with fixed $d>0$, then

$$
P(X \in[x, x+d])=\int_{x}^{x+d} \frac{1}{b-a} d t=\frac{d}{b-a},
$$

which is independent of $x$. This fact motivates the distribution's name. Restricting $(a, b)=(0,1)$, the resulting distribution $R(0,1)$ is called a standard rectangular distribution or a square distribution.

\section{X-Folding of a rectangular distribution and its properties}

In this section we will start introducing the definition of the X-Folding of the corresponding rectangular with unit area of the $R(a, b)$ distribution. Here, we will discuss this folding and its corresponding areas, distributions and properties.

Definition 3.1. The X-Folding on the $R(a, b)$ distribution is the map $F_{X}: R^{2} \rightarrow$ $R^{2}$ such that:

$$
F_{X}(x, y)=\left\{\begin{array}{lll}
\left(2 \mu_{X}-x, y\right) & : & a \leq x \leq \mu_{X} \\
(x, y) & : & \mu_{X}<x \leq b \\
0 & : & \text { otherwise }
\end{array}\right.
$$

where $\mu_{X}$ is the corresponding mean of $R(a, b)$ distribution.

Definition 3.2. A set of singular points of the X-Folding $F_{X}$ on $R(a, b)$ is denoted by $\sum F_{X}$ and it is defined by $\sum F_{X}=\left\{\left(\mu_{X}, y\right) \in R^{2}: y \in\left[0, \frac{1}{b-a}\right]\right\}$.

Definition 3.3. Let $a_{X_{k}}=\frac{a+\left(2^{k}-1\right) b}{2^{k}}$ and let $\mu_{X_{k}}$ be the corresponding mean of $R\left(a_{X_{k}}, b\right)$ distribution, $k=0,1,2,3, \ldots$. The sequence $\left\{F_{X_{k}}\right\}_{k=0}^{\infty}$ of the X-Folding on the $R(a, b)$ distribution is defined by:

$$
F_{X_{k}}(x, y)=\left\{\begin{array}{ccc}
\left(2 \mu_{X_{k}}-x, y\right) & : & a_{X_{k}} \leq x \leq \mu_{X_{k}} \\
(x, y) & : & \mu_{X_{k}}<x \leq b \\
0 & : & \text { otherwise }
\end{array}\right.
$$


with $\left.\sum F_{X_{k}}=\left\{\left(\mu_{X_{k}}, y\right) \in R^{2}\right\}: y \in\left[0, \frac{1}{b-a}\right]\right\}$ as a corresponding set of singular points of $F_{X_{k}}$.

Lemma 3.1. Let $\left\{F_{X_{k}}\right\}_{k=0}^{\infty}$ be a sequence of the $X$-Folding on the $R(a, b)$ distribution. Then the sequence of the corresponding areas $\left\{A_{X_{k}}\right\}_{k=0}^{\infty}$ of $\left\{F_{X_{k}}\right\}_{k=0}^{\infty}$ has the following properties:

(1) $A_{X_{k}}=F_{X_{k}}\left(A_{X_{k-1}}\right)=\frac{1}{2^{k}}, k=1,2,3, \ldots$, with $A_{X_{0}}=1$,

(2) the sequence $\left\{A_{X_{k}}\right\}_{k=0}^{\infty}$ is a monotone decreasing sequence of areas,

(3) $\lim _{k \rightarrow \infty} A_{X_{k}}=0$ and $\sum_{k=1}^{\infty} A_{X_{k}}=A_{X_{0}}$.

Proof. Follows directly by using the Definition 3.3 and the sum of a geometric sequence.

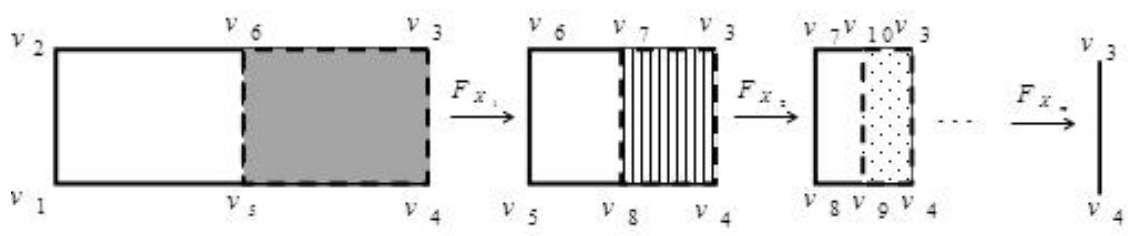

Figure 1: The corresponding areas of a sequence of the X-Folding on the $R(a, b)$ distribution.

Figure 1 illustrates the corresponding generated areas by applying a sequence of the $\mathrm{X}$-Folding on the $R(a, b)$ distribution. The vertices in this figure are given by the following:

$$
\begin{gathered}
\nu_{1}=(a, 0), \nu_{2}=\left(a, \frac{1}{b-a}\right), \nu_{3}=\left(b, \frac{1}{b-a}\right), \nu_{4}=(b, 0), \nu_{5}=\left(\frac{a+b}{2}, 0\right), \\
\nu_{6}=\left(\frac{a+b}{2}, \frac{1}{b-a}\right), \nu_{7}=\left(\frac{a+3 b}{4}, \frac{1}{b-a}\right), \nu_{8}=\left(\frac{a+3 b}{4}, 0\right), \nu_{9}=\left(\frac{a+7 b}{8}, 0\right), \ldots
\end{gathered}
$$

Lemma 3.2. Let $X=X_{0}$ be a continuous random variable which has the $R(a, b)$ distribution with the minimum value a in $X$-axes. Apply a sequence of $X$-Folding $\left\{F_{X_{k}}\right\}_{k=0}^{\infty}$ defiened on the $R(a, b)$ distribution, then we get on a sequence of the corresponding minimum values $\left\{a_{X_{k}}\right\}_{k=0}^{\infty}=\left\{\frac{a+\left(2^{k}-1\right) b}{2^{k}}\right\}_{k=0}^{\infty}$ of the generated intervals on $X$-axes.

Proof. Follows directly by applying Definition 3.3 and also by using the mathematical induction.

Proposition 3.1. Let $X=X_{0}$ be a continuous random variable which has the $R(a, b)$ distribution with the minimum value a in $X$-axes. Applying a sequence of $X$-Folding $\left\{F_{X_{k}}\right\}_{k=0}^{\infty}$ defined on the $R(a, b)$ distribution. Then, a sequence of continuous random variables $\left\{X_{k}\right\}_{k=0}^{\infty}$ of the $k$-th $X$-Folding $F_{X_{k}}$ can be generated with 
the following properties:

(1) Each random variable $X_{k}, k=0,1,2,3, \ldots$, has rectangular distribution on the corresponding interval $\left(a_{X_{k}}, b\right), k=0,1,2,3, \ldots$, and the probability density function is given by the following closed form:

$$
g_{X_{k}}(x)=\left\{\begin{array}{clc}
\frac{2^{k}}{b-a} & : & x \in\left[a_{X_{k}}, b\right] \\
0 & : & \text { otherwise }
\end{array}\right.
$$

(2) the mean $\mu_{X_{k}}$ of $X_{k} ; k=0,1,2,3, \ldots$, is given by:

$$
\mu_{X_{k}}=\frac{a+\left(2^{k+1}-\right) b}{2^{k+1}} ; k=0,1,2,3, \ldots,
$$

(3) the variance of $X_{k} ; k=0,1,2,3, \ldots$, is given by:

$$
\sigma_{X_{k}}^{2}=\frac{1}{2^{2 k}} \frac{(b-a)^{2}}{12} ; k=0,1,2,3, \ldots,
$$

(4) the cumulative distribution function of $X_{k}, k=0,1,2,3, \ldots$, is given by:

$$
G_{X_{k}}(x)=\left\{\begin{array}{ccc}
0 & : \quad x \leq a_{X_{k}} \\
\frac{(x-a)+\left(2^{k}-1\right)(x-b)}{b-a} & : & a_{X_{k}}<x<b \\
1 & : & x \geq b
\end{array}\right.
$$

(5) the moment-generating function of $X_{k}, k=0,1,2,3, \ldots$, is given by:

$$
M_{X_{k}}(t)=\frac{e^{b t}-e^{a_{X_{k}}} t}{\left(b-a_{X_{k}}\right) t}, \quad|t| \neq 0
$$

(6) the characteristic function of the random variable $X_{k}, k=0,1,2,3, \ldots$, is given by:

$$
\Psi_{X_{k}}(t)=\frac{e^{i b t}-e^{i a_{X_{k}} t}}{i\left(b-a_{X_{k}}\right) t}, \quad|t| \neq 0, \quad i=\sqrt{-1} .
$$

Proof. (1) By applying the Folding $F_{X_{1}}$ of the rectangular with area $A_{X_{0}}$ on the interval $(a, b)$ on $\mathrm{X}$-axes, a continuous rectangular random variable $X_{1}$ can be generated on the interval $\left(a_{X_{1}}, b\right)$. The corresponding probability density function of $X_{1}$ is given in the following closed form:

$$
g_{X_{1}}(x)=\left\{\begin{array}{cll}
\frac{2}{b-a} & : & x \in\left[a_{X_{1}}, b\right] \\
0 & : & \text { otherwise }
\end{array}\right.
$$


Repeat the previous step by the Folding $F_{X_{2}}$ with respect to the obtained area $A_{X_{1}}=F_{X_{1}}\left(A_{X_{0}}\right)$, then $X_{2}$ can be generated on the interval $\left(a_{X_{2}}, b\right)$ with the corresponding probability density function in the following closed form:

$$
g_{X_{2}}(x)=\left\{\begin{array}{ccc}
\frac{2^{2}}{b-a} & : & x \in\left[a_{X_{2}}, b\right] \\
0 & : & \text { otherwise }
\end{array}\right.
$$

We iterate the process as in the previous steps by apply $F_{X_{k}}, k=3,4,5, \ldots$, with respect to $A_{X_{k}}=F_{X_{k}}\left(A_{X_{k-1}}\right)$, respectively. It is easy to get on a corresponding sequence of continuous random variables $\left\{X_{k}\right\}_{k=0}^{\infty}$ of $\left\{F_{X_{k}}\right\}_{k=0}^{\infty}$ with (3.3) as a closed form of the probability density function.

(2) Due to Definition 3.3 and the definition of the mean, we will get on a sequence of corresponding mean $\mu_{X_{k}}$ of the random variable $X_{k}, k=0,1,2,3, \ldots$, as in (3.4).

(3) Since each random variable $X_{k}$ has $R\left(a_{X_{k}}, b\right)$ distribution, $k=0,1,2,3, \ldots$, then its variance is given by:

$$
\sigma_{X_{k}}^{2}=\frac{\left(b-a_{X_{k}}\right)^{2}}{12} ; k=0,1,2,3, \ldots
$$

It is a direct consequence of the last equation and Lemma 3.1 that, the closed form (3.5) of the variance $\sigma_{X_{k}}^{2}$ of $X_{k}, k=0,1,2,3, \ldots$, can be obtained.

(4) Since each random variable $X_{k}$ has $R\left(a_{X_{k}}, b\right)$ distribution, $k=0,1,2,3, \ldots$, then its corresponding cumulative distribution function $G_{X_{k}}, k=0,1,2,3, \ldots$, in the closed form (3.6) can be easily deduced by replacing $a$ with $a_{X_{k}}$ in the form (2.2).

(5) Putting $a_{X_{k}}$ instead of $a$ in (2.4), then the closed form (3.7) of the corresponding moment-generating function of $X_{k}, k=0,1,2,3, \ldots$, can be also easily obtained.

(6) As in (5), replace $a$ by $a_{X_{k}}$ in (2.4) then the corresponding characteristic function of $X_{k}, k=0,1,2,3, \ldots$, is given by the closed form (3.8).

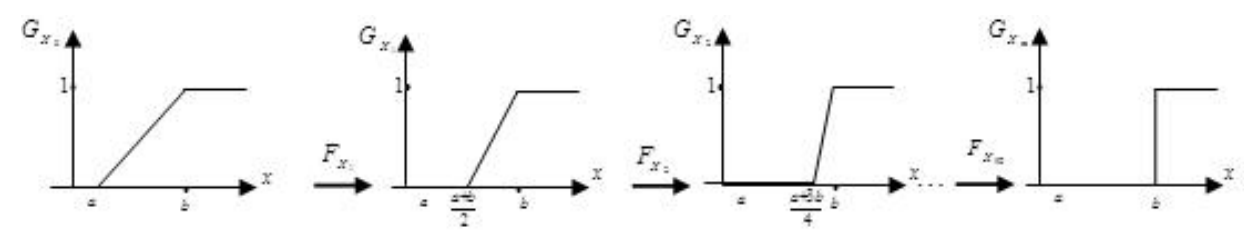

Figure 2: The corresponding cumulative distribution functions of a sequence $\left\{F_{X_{k}}\right\}_{k=0}^{\infty}$. 
Figure 2 illustrates the corresponding generated cumulative distribution functions by applying a sequence of the X-Folding on the $R(a, b)$ distribution.

Proposition 3.2. Let $\left\{X_{k}\right\}_{k=0}^{\infty}$ be a generated sequence of continuous random variables of the sequence $\left\{F_{X_{k}}\right\}_{k=0}^{\infty}$ of the $k$-th $X$-Folding, where $X=X_{0}$ is the $R(a, b)$ variable, and let $\left\{A_{X_{k}}\right\}_{k=0}^{\infty}$ be a sequence of the corresponding areas. Then, for $k=0,1,2,3, \ldots$, we can find the following statements:

(1) $P_{X_{k}}\left(a_{X_{k}} \leq X \leq b\right)=P_{X}(a \leq X \leq b)=1$,

(2) $A_{X_{k}}=P_{X}\left(a_{X_{k}} \leq X \leq b\right)=\frac{1}{2^{k}}$,

(3) $P_{X}\left(a_{X_{k}} \leq X \leq b\right)=\frac{1}{2^{k}} P_{X_{k}}\left(a_{X_{k}} \leq X \leq b\right)$,

(4) for any two real constants $c$ and $d, P_{X}(c \leq X \leq d)=\frac{1}{2^{k}} P_{X_{k}}(c \leq X \leq d)$.

Proof. It is a direct consequence of the previous definitions, lemmas and proposition that the relations (1) - (4) can be easily derived.

Corollary 3.1. Let $\left\{X_{k}\right\}_{k=0}^{\infty}$ be a generate sequence of continuous random variables of the sequence $\left\{F_{X_{k}}\right\}_{k=0}^{\infty}$ of the $k$-th $X$-Foldings, where $X=X_{0}$ is the $R(a, b)$ variable, and let $\left\{A_{X_{k}}\right\}_{k=0}^{\infty}$ be a sequence of the corresponding areas. Then, for $k=0,1,2,3, \ldots$, we can find the following statements:

(1) $A_{X_{k}}=\frac{1}{2^{k}} A_{X_{0}}$,

(2) $g_{X_{k}}(x)=2^{k} g_{X_{0}}(x)$,

(3) $\mu_{X_{k}}=\frac{1}{2^{k}}\left[\mu_{X_{0}}+\left(2^{k}-1\right) b\right]$,

(4) $\sigma_{X_{k}}^{2}=\frac{1}{2^{k}} \sigma_{X_{0}}^{2}$,

(5) $G_{X_{k}}(x)=2^{k}\left[G_{X_{0}}(x)-1\right]+1 ; a_{X_{k}}<x<b$.

Proof. The relations (1), (2), (3), (4) and (5) can be easily obtained from Lemma 3.1, Lemma 3.2, and the equations (3.3), (3.4), (3.5), (3.6).

\section{Y-Folding of a rectangular distribution and its properties}

According to the presented definitions, lemmas, propositions and corollary in section 3, we will define the Y-Folding with respect to the $R\left(0, \frac{1}{b-a}\right)$ distribution, which is equivalent to the $R(a, b)$ distribution. We will give some results with respect to the Y-Folding, which are similar to the discussed previous results with respect to the X-Folding. According to this mentioned similarity, here we omit the proofs. 
Definition 4.1. The Y-Folding on the $R\left(0, \frac{1}{b-a}\right)$ distribution is the map $F_{Y}: R^{2} \rightarrow R^{2}$ such that:

$$
F_{Y}(x, y)=\left\{\begin{array}{lcc}
\left(x, 2 \mu_{Y}-y\right) & : & 0 \leq y \leq \mu_{y} \\
(x, y) & : & \mu_{Y}<y \leq \frac{1}{b-a} \\
0 & : & \text { otherwise }
\end{array}\right.
$$

where $\mu_{Y}$ is the corresponding mean of $R\left(0, \frac{1}{b-a}\right)$ distribution.

Definition 4.2. A set of singular points of the Y-Folding $F_{Y}$ on $R\left(0, \frac{1}{b-a}\right)$ is denoted by $\sum F_{Y}$ and it is defined by $\sum F_{Y}=\left\{\left(x, \mu_{Y}\right) \in R^{2} \quad: \quad x \in[a, b]\right\}$.

Definition 4.3. Let $a_{Y_{k}}=\frac{2^{k}-1}{2^{k}(b-a)}$ and let $\mu_{Y_{k}}$ be the corresponding mean of $R\left(a_{Y_{k}}, \frac{1}{b-a}\right)$ distribution, $k=0,1,2,3, \ldots$. The sequence $\left\{F_{Y_{k}}\right\}_{k=0}^{\infty}$ of the YFolding on the $R\left(0, \frac{1}{b-a}\right)$ distribution is defined by:

$$
F_{Y_{k}}(x, y)=\left\{\begin{array}{ccc}
\left(x, 2 \mu_{Y_{k}}-y\right) & : & a_{Y_{k}} \leq y \leq \mu_{Y_{k}} \\
(x, y) & : & \mu_{Y_{k}}<y \leq \frac{1}{b-a} \\
0 & : & \text { otherwise }
\end{array}\right.
$$

with $\sum F_{Y_{k}}=\left\{\left(x, \mu_{Y_{k}}\right) \in R^{2}: x \in[a, b]\right\}$ as a corresponding set of singular points of $F_{Y_{k}}$.

Lemma 4.1. Let $\left\{F_{Y_{k}}\right\}_{k=0}^{\infty}$ be a sequence of the $Y$-Folding on the $R\left(0, \frac{1}{b-a}\right)$ distribution. Then the sequence of the corresponding areas $\left\{A_{Y_{k}}\right\}_{k=0}^{\infty}$ of $\left\{F_{Y_{k}}\right\}_{k=0}^{\infty}$ has the following properties:

(1) $A_{Y_{k}}=F_{Y_{k}}\left(A_{Y_{k-1}}\right)=\frac{1}{2^{k}}, k=1,2,3, \ldots$, with $A_{Y_{0}}=1$,

(2) the sequence $\left\{A_{Y_{k}}\right\}_{k=0}^{\infty}$ is a monotone decreasing sequence of areas,

(3) $\lim _{k \rightarrow \infty} A_{Y_{k}}=0$ and $\quad \sum_{k=1}^{\infty} A_{Y_{k}}=A_{Y_{0}}$.

Lemma 4.2. Let $Y=Y_{0}$ be a continuous random variable which has the $R\left(0, \frac{1}{b-a}\right)$ distribution with the minimum value zero in $Y$-axes. Apply a sequence of $Y$-Folding $\left\{F_{Y_{k}}\right\}_{k=0}^{\infty}$ defined on the $R\left(0, \frac{1}{b-a}\right)$ distribution, then we get on a sequence of the corresponding minimum values $\left\{a_{Y_{k}}\right\}_{k=0}^{\infty}=\left\{\frac{2^{k}-1}{2^{k}(b-a)}\right\}_{k=0}^{\infty}$ of the generated intervals on $Y$-axes.

Proposition 4.1. Let $Y=Y_{0}$ be a continuous random variable which has the $R\left(0, \frac{1}{b-a}\right)$ distribution with the minimum value zero in $Y$-axes. Applying a sequence of $Y$-Folding $\left\{F_{Y_{k}}\right\}_{k=0}^{\infty}$ defined on the $R\left(0, \frac{1}{b-a}\right)$ distribution. Then, a sequence of 
continuous random variables $\left\{Y_{k}\right\}_{k=0}^{\infty}$ of the $k$-th $Y$-Folding $F_{Y_{k}}$ can be generated with the following properties:

(1) Each $Y_{k}, k=0,1,2,3, \ldots$, has rectangular distribution on the corresponding interval $\left(a_{Y_{k}}, \frac{1}{b-a}\right), k=0,1,2,3, \ldots$, and the probability density function is given by the following closed form:

$$
g_{Y_{k}}(y)=\left\{\begin{array}{ccc}
2^{k}(b-a) & : & y \in\left[a_{Y_{k}}, \frac{1}{b-a}\right] \\
0 & : & \text { otherwise }
\end{array}\right.
$$

(2) the mean $\mu_{Y_{k}}$ of $Y_{k} ; k=0,1,2,3, \ldots$, is given by:

$$
\mu_{Y_{k}}=\frac{2^{k+1}-1}{2^{k+1}(b-a)} ; k=0,1,2,3, \ldots,
$$

(3) the variance of $Y_{k} ; k=0,1,2,3, \ldots$, is given by:

$$
\sigma_{Y_{k}}^{2}=\frac{1}{2^{2 k}} \frac{1}{12(b-a)^{2}} ; k=0,1,2,3, \ldots,
$$

(4) the cumulative distribution function of $Y_{k}, k=0,1,2,3, \ldots$, is given by:

$$
G_{Y_{k}}(y)=\left\{\begin{array}{clc}
0 & : & y \leq a_{Y_{k}} \\
2^{k}(b-a) y+\left(2^{k}-1\right) & : & a_{Y_{k}}<y<\frac{1}{b-a} \\
1 & : & y \geq \frac{1}{b-a}
\end{array}\right.
$$

(5) the moment-generating function of $Y_{k}, k=0,1,2,3, \ldots$, is given by:

$$
M_{Y_{k}}(t) \frac{e^{\frac{1}{b-a} t}-e^{a_{Y_{k}} t}}{\left(\frac{1}{b-a}-a_{Y k}\right) t}, \quad|t| \neq 0,
$$

(6) the characteristic function of the random variable $Y_{k}, k=0,1,2,3, \ldots$, is given by:

$$
\Psi_{Y_{k}}(t)=\frac{e^{\frac{i}{b-a} t}-e^{i a_{Y_{k}} t}}{i\left(\frac{1}{b-a}-a_{Y k}\right) t}, \quad|t| \neq 0, \quad i=\sqrt{-1} .
$$

Proposition 4.2. Let $\left\{Y_{k}\right\}_{k=0}^{\infty}$ be a generated sequence of continuous random variables of the sequence $\left\{F_{Y_{k}}\right\}_{k=0}^{\infty}$ of the $k$-th $X$-Folding, where $Y=Y_{0}$ is the $R\left(0, \frac{1}{b-a}\right)$ variable, and let $\left\{A_{Y_{k}}\right\}_{k=0}^{\infty}$ be a sequence of the corresponding areas. Then, for $k=0,1,2, \ldots$, we can find the following statements: 
(1) $P_{Y_{k}}\left(a_{Y_{k}} \leq Y \leq \frac{1}{b-a}\right)=P_{Y}\left(0 \leq Y \leq \frac{1}{b-a}\right)=1$,

(2) $A_{Y_{k}}=P_{Y}\left(a_{Y_{k}} \leq Y \leq \frac{1}{b-a}\right)=\frac{1}{2^{k}}$

(3) $P_{Y}\left(a_{Y_{k}} \leq Y \leq \frac{1}{b-a}\right)=\frac{1}{2^{k}} P_{Y_{k}}\left(a_{Y_{k}} \leq Y \leq \frac{1}{b-a}\right)$,

(4) for any two real constants $c$ and $d, P_{Y}(c \leq Y \leq d)=\frac{1}{2^{k}} P_{Y_{k}}(c \leq Y \leq d)$.

Corollary 4.1. Let $\left\{Y_{k}\right\}_{k=0}^{\infty}$ be a generated sequence of continuous random variables of the sequence $\left\{F_{Y_{k}}\right\}_{k=0}^{\infty}$ of the $k$-th $Y$-Foldings, where $Y=Y_{0}$ is the $R\left(0, \frac{1}{b-a}\right)$ variable, and let $\left\{A_{Y_{k}}\right\}_{k=0}^{\infty}$ be a sequence of the corresponding areas. Then, for $k=0,1,2, \ldots$, we can find the following statements:

(1) $A_{Y_{k}}=\frac{1}{2^{k}} A_{Y_{0}}$

(2) $g_{Y_{k}}(y)=2^{k} g_{Y_{0}}(y)$,

(3) $\mu_{Y_{k}}=\frac{1}{2^{k}}\left[\mu_{Y_{0}}+\frac{2^{k}-1}{b-a}\right]$,

(4) $\sigma_{Y_{k}}^{2}=\frac{1}{2^{k}} \sigma_{Y_{0}}^{2}$,

(5) $G_{Y_{k}}(y)=2^{k}\left[G_{Y_{0}}(y)-1\right]+1 ; a_{Y_{k}}<y<\frac{1}{b-a}$.

\section{Application, [11]}

Let the continuous random variable $X$ denote the current measured in a thin copper wire in milliamperes " $m A$ ". Assume that the range of $X$ in the interval $[0,20 \mathrm{~mA}]$, and assume that the probability density function of $X$ is $g(X)=0.05$ for $0 \leq x \leq 20$. It is assumed that $g(X)=0.05$ whenever it is not specially defined. For the copper current measurement, the cumulative distribution function of $X$ is given by

$$
G(x)=\left\{\begin{array}{ccc}
0 & : & x \leq 0 \\
0.05 x & : & 0<x<20 \\
1 & : & 20 \leq x
\end{array}\right.
$$

It is clear that the variable $X$ has the $R(0,20)$ distribution with mean $\mu=10 \mathrm{~mA}$, variance $\sigma^{2}=33.33 \mathrm{~mA}^{2}$ and standard deviation $\sigma=5.77 \mathrm{~mA}$. Notice that in the definition of $G(x)$ any "<" can be changed to " $\leq$ " and vice versa. That is, $G(x)$ can be defined as either $0.05 x$ or 0 , and $G(x)$ can be defined as either $0.05 x$ or 1 at the end-point $x=20$. Apply a sequence of the X-Folding on the $R(0,20)$ distribution, we get on a thin copper wire with high resistance. 
Figure 3 illustrates the corresponding generated probability density functions by applying $F_{X_{0}}, F_{X_{1}}, F_{X_{2}}, F_{X_{3}}, F_{X_{4}}$ iteratively in the X-direction on the given $R(0,20)$ distribution.

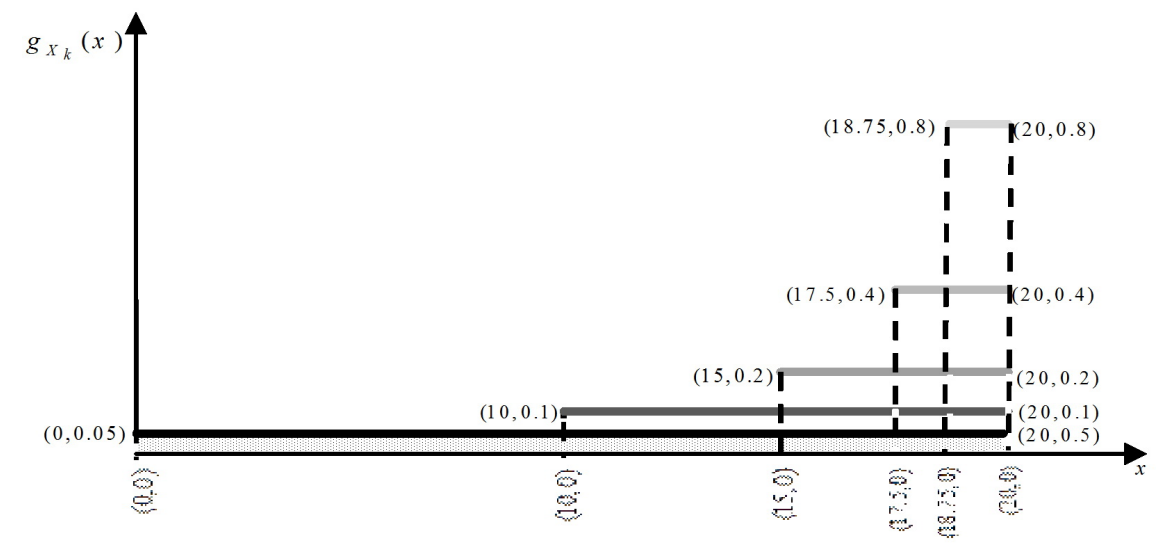

Figure 3: Corresponding Probability density functions of the X-Folding for the $R(0,20)$ distribution.

The probability that a current measurement is less than $10 \mathrm{~mA}$ can be obtained as

$$
P_{X}(X<10)=\frac{1}{2^{k}} P_{X_{k}}(X<10)=0.5 ; \quad k=0,1,2,3, \ldots,
$$

the probability that a measurement of current is between 5 and $10 \mathrm{~mA}$ is

$$
P_{X}(5<X<10)=\frac{1}{2^{k}} P_{X_{k}}(5<X<10)=0.25 ; \quad k=0,1,2,3, \ldots,
$$

and also the probability that a measurement of current is between 5 and $15 \mathrm{~mA}$ is

$$
P_{X}(5<X<15)=\frac{1}{2^{k}} P_{X_{k}}(5<X<15)=0.5 ; \quad k=0,1,2,3, \ldots .
$$

All results in section 3 can be obtained with respected to the $R(0,20)$ distribution by replacing $(a, b)$ by $(0,20)$.

\section{Conclusion}

We defined the foldings in X-direction and in Y-direction which are denoted by the X-Folding and the Y-Folding respectively. We applied this folding on the area $P(a \leq X \leq b)$, where $X$ has $R(a, b)$ distribution with the parameters $a, b$. A family of the corresponding rectangular probability distributions is generated. Some closed forms of the corresponding start-points, probability density functions, cumulative distribution functions, means, variances, moments-generating functions 
and characteristic functions of the applied foldings in each direction were derived. Some properties of the generated areas from the used sequence of foldings were discussed. We obtained a recurrence relation to calculate the probability either by the original rectangular or by each of a generated sequence of distributions. We presented a physical application of the defined folding. Our future work will be devoted to applying the folding on other probability distribution and also we will tray to illustrate iteratively folding in the two directions. Also, we will look for general interesting continuous probability distributions.

\section{References}

[1] M. Basher, On the Folding of Finite Topological Space, International Mathematical Forum, 7(2012), 745-752.

[2] P. Di-Francesco, Folding and coloring problem in mathematics and physics, Bulletin of the American Mathematics Society, 37(2000), 251-307.

[3] M. El-Ghoul, Folding of manifolds, Ph.D. Thesis, Tanta Univ., Egypt, (1985).

[4] M. El-Ghoul and M. E. Basher, The Invariant of Immersions under Isotwist Folding, 46(2006), 139-144.

[5] M. El-Ghoul, S. I. Nada and R. M. Elanin, On the folding of rings, International Journal of Algebra, 3 10(2009), 475-482.

[6] E. El-Kholy, Isometric and topological folding of manifolds, Ph.D. Thesis, University of Southampton, UK, (1981).

[7] E. El-Kholy and A. El-Esawy , Graph folding of some special graphs, Journal of Mathematics and Statistics, 1(2005), 66-70.

[8] E. El-Kholy and M. El-Ghoul, Simplicial foldings, Journal of the Faculty of Education, Tanta Univ., Egypt, 18(1993), 443-455.

[9] E. El-Kholy and R. M. Shahin, Cellular folding, Journal of Institute of Math \& Comp. Sci., 3(1998), 177-181.

[10] J. E. Freund, I. Miller and M. Miller, Mathematical Statistics with Applications, 7th ed. Prentice Hall PTR, (2003).

[11] D. C. Montgomery and G. C. Runger, Applied Statistics and Probability for Engineers, 2nd ed. John Wiley \& Sons, Inc. New York, (1999).

[12] S. I. Nada and E. H. Mamunda, On the folding of graphs-theory and applications, Chaos, Solitons \& Fractals, 42(2009), 669-675.

[13] S. A. Robertson, Isometric folding of Riemannian manifolds, Proceeding of the Royal Society of Edinburgh, 79:3-4(1977), 275-284. 